\title{
tic\&société
}

Vol. 9, No 1-2 | 1 er semestre 2015 - 2ème semestre 2015

Les religions au temps du numérique

\section{Multimédiatisation et événement religieux : le cas de la campagne d'évangélisation l'« Horizon de l'espérance » de Hope Channel Romania (Speranta TV)}

\section{Mihaela Alexandra TUDOR}

\section{(2) OpenEdition Journals}

Electronic version

URL: http://journals.openedition.org/ticetsociete/1840

DOI: 10.4000/ticetsociete. 1840

\section{Publisher}

Association ARTIC

\section{Electronic reference}

Mihaela Alexandra TUDOR, « Multimédiatisation et événement religieux

le cas de la campagne d'évangélisation l'« Horizon de l'espérance » de Hope Channel Romania

(Speranta TV) », tic\&société [Online], Vol. 9, № 1-2 I 1er semestre 2015 - 2ème semestre 2015, Online since 08 January 2016, connection on 19 April 2019. URL : http://journals.openedition.org/ ticetsociete/1840 ; DOI : 10.4000/ticetsociete.1840 
tic\&société - 9 (1-2), 2015

Multimédiatisation et événement religieux : le cas de la campagne d'évangélisation l' "Horizon de l'espérance " de Hope Channel Romania (Speranta TV)

Mihaela Alexandra TUDOR

Université Paul-Valéry Montpellier 3

ORC larsic - Corhis (EA 4700)

Route de Mende, 34199 Montpellier Cedex 5

mihaela.tudor.com@gmail 
Multimédiatisation et événement religieux

\section{Mihaela Alexandra TUDOR}

\section{Multimédiatisation et événement religieux : le cas de la campagne d'évangélisation I" « Horizon de l'espérance » de Hope Channel Romania (Speranta TV)}

Mihaela Alexandra TUDOR est maître de conférences habilitée à diriger des recherches en sciences de l'information et de la communication à l'Université Paul Valéry de Montpellier. Elle est directrice de rédaction de la revue Essachess - Journal for Communication Studies et responsable de la coopération internationale et du programme des projets stratégiques du réseau international ORC larsic-Corhis (EA 4700). Ses recherches sur les fondements épistémologiques des sciences de l'information et de la communication qui font l'objet de nombreux articles et communications internationales portent principalement sur la communication symbolique et la communication de la foi à travers les (nouveaux) médias.

Résumé : Dans cet article nous questionnons les formes d'engagement des médias religieux avec les nouvelles technologies de l'information et de la communication (NTIC) pour "évangéliser» en reconstituant le chemin fait par la chaîne de télévision néo-protestante Hope Channel Romania (Speranta TV) pour la mise en œuvre de la campagne d'évangélisation l' «Horizon de l'espérance». Si l'on considère que les NTIC ne sont pas encore stabilisées, l'hypothèse mise à l'épreuve ici consiste à montrer que le produit d'évangélisation multimédia créé par Speranta TV s'inscrit davantage dans la logique de la convergence cross-media des médias traditionnels et des nouveaux médias que dans la logique du « transfert d'autorité » vers les nouveaux médias.

Mots-clés : évangélisation, convergence cross-media, médias néo-protestants, nouveaux médias.

Abstract: Multi-mediatization and religious event: the case of the evangelical campaign "Horizon of Hope" on Hope Channel 


\section{Mihaela Alexandra TUDOR}

Romania (Speranta TV) - In this article we will question how the religious media engages with the new Information and Communication Technologies (ICTs) for evangelization by reconstructing neo-protestant Hope Channel Romania's (Speranta TV) work to implement the evangelical "Horizon of Hope" campaign. Considering that ICTs cannot be regarded yet as stabilized, we suggest that the product of media evangelization created by this religious media is rooted in the logic of cross media convergence between old and new media rather than in a logic of transfer of authority toward the new media.

Keywords: evangelization, convergence cross media, neoProtestant media, new media.

Resumen: Multi-mediatización y evento religioso: el caso de la campaña evangelistica "El horizon de la esperanza Hope Channel Rumania (Speranta TV) - En este artículo estudia como los medios religiosos utilizan las nuevas tecnologías de la información y de la comunicación (NTIC)- informatica de la evangelizacion para evangelizar y analiza para reconstruir el camino abierto por la cadena de televisión neoprotestante La Voz de la Esperanza Rumana (Speranta TV) para introducir la campaña evangelista "El horizonte de la esperanza". Considerando que las NTIC no están aún establizadas, la hipótesis que aquí se propone es mostrar que el producto evangelistico creado por Speranta TV se inscribe más en la lógica de convergencia cross-media entre los medios de comunicación tradicionales y los nuevos medios que en la lógica de "transferencia de autoridad" hacia los nuevos medios de comunicación.

Palabras clave: evangelización, convergencia cross-media, neo-protestantes, medios de comunicación, nuevos medios de comunicación.

Comment les médias religieux traditionnels des mouvements néo-protestants se sont-ils appropriés internet et plus particulièrement les médias sociaux dans leurs efforts 
Multimédiatisation et événement religieux

concernant la transmission de leurs enseignements et de leurs messages? Comment les campagnes d'évangélisation télévisées ont évolué et ont été redéfinies face à la révolution numérique? En quoi ces campagnes sont-elles devenues innovantes face à internet et aux médias sociaux ? S'agit-il de faire un travail pédagogique de diffusion de l'évangile par la mise en réseau des médias (cross-media) ou par l'usage des différents médias ou hors médias dans la logique de communication transmedia?

Dans cet article, nous allons tenter d'apporter des éléments de réponse par le biais d'une étude de cas. Il en résulte que les campagnes télévisées d'évangélisation du mouvement néoprotestant adventiste prennent une forme d'engagement spécifique à travers les NTIC en mettant en place des dispositifs interactifs censés faciliter le glissando de la transmission de l'évangile à la communication de l'évangile avec des compétences et logiques étendues.

L'étude de cas que nous allons présenter est fondée sur l'analyse de Hope Channel Romania (Speranta TV), média religieux néo-protestant adventiste ${ }^{1}$ faisant partie du réseau mondial Hope Channel, pour la mise en place de la campagne d'évangélisation l' « Horizon de l'espérance » qui a eu lieu entre le 6 et le 14 décembre 2013. L'explication de l'impact de la campagne d'évangélisation repose principalement sur le processus d'émancipation des supports et des médias classiques. D'autre part, la mise en place d'un dispositif mobilisant, simultanément, les médias traditionnels et les nouveaux médias (old media et new media) fait valoir le rôle croissant de la communication dans la transmission de la foi dans le contexte de l'émergence rapide des NTIC.

\footnotetext{
${ }^{1}$ Les médias néo-protestants sont les institutions médiatiques affiliées aux églises néo-protestantes (historiquement issues des grands cultes protestants) tels que les baptistes, les adventistes, les mormons, les pentecôtistes, etc.
} 


\section{Mihaela Alexandra TUDOR}

\section{Perspectives théoriques}

\subsection{Médias, nouveaux médias et religion}

Depuis soixante-dix ans d'étude des médias traditionnels, nous avons déjà une idée claire des objets étudiés: la communication média, les institutions et organisations médias (presse écrite, radio, télévision, etc.), les productions matérielles et culturelles de ces institutions et organisations, etc. Depuis la publication de l'ouvrage de Marshall McLuhan Understanding Media : the Extension of Man en 1964, on est de plus en plus conscient des changements rapides et dramatiques des catégories et modalités de communication, des problèmes théoriques, philosophiques, sociologiques, économiques, etc., soulevés par l'apparition et l'essor des médias (De Vries, 2001). Ces directions de recherches ont constitué la préoccupation centrale de plusieurs études : celles de Theodor Adorno et de Max Horkheimer (2002) sur les médias et la culture de masse, de Paul Lazarsfeld et d'Elihu Katz (1995/2008) sur les effets des médias sur la société, de Pierre Bourdieu (1996) sur la télévision, de Jean-Luc Marion (1991) sur la phénoménologie de l'image, de Manuel Castells (1999) sur les technologies et la société d'information ou de Jérôme Bourdon (1997 [2009]) sur les médias de masse et interpersonnels. Même si certaines de ces contributions fondamentales se sont intéressées de manière indirecte à la religion et à la médiatisation (Stout, 2011) - en les intégrant à la notion de culture ou d'identité politique, à la propagande ou à la mémoire... -, elles ont permis le cadrage scientifique nécessaire à la compréhension des interactions et des rapports entre la religion et les médias.

Dans le contexte particulier de l'essor des NTIC, qui a fait des médias un champ en constante mutation, l'intérêt des scientifiques pour le sacré et le religieux sur «la toile» commence à devenir manifeste dans la deuxième moitié des années 1990. O'Leary (1996) et Brasher (1996), pionniers dans l'étude de la communication médiatisée par l'ordinateur computer-mediated communication, ont analysé la manière dont la religion et le sacré sont influencés par l'environnement 
Multimédiatisation et événement religieux

en ligne. Les problématiques de recherche ont alors connu une diversification rapide couvrant des thématiques comme l'éthique du religieux et la réalité virtuelle (Houston, 1998), les modalités par lesquelles les individus et le sacré sont mis en relation à travers la technologie (Cobb, 1998 ; Wertheim, 1999), les adaptations en ligne des pratiques traditionnelles du religieux (Zaleski, 1997). Ces recherches initiales ont tenté de définir et catégoriser les phénomènes du sacré dans le World Wilde Web et de construire la distinction théorique entre religion online, comme forme traditionnelle de religion mise en ligne, et online religion, comme religion adaptée à internet pour créer de nouvelles formes d'interactions spirituelles en réseau (Helland, 2000).

Récemment, au début du $21^{\mathrm{e}}$ siècle, la recherche portant sur le religieux et notamment sur le sacré dans le World Wilde Web s'est diversifiée davantage avec le développement des études sur la « société de l'information ». La plupart des études sur le sacré et la religion sur internet ont emprunté le même trajet que les études et les recherches portant généralement sur la culture et la « vie » en ligne. Leurs débuts rhapsodiques qui glorifiaient ou «diabolisaient» les nouvelles possibilités de «faire religion » dans la vie virtuelle ont laissé place à la recherche empirique (Dawson, Cowan, 2004). Dans cette optique d'émergence, Heidi Campbell a inventorié quatre catégories de recherche portant sur le sacré et le religieux (Campbell, 2003, 2006). La première catégorie consiste en l' « analyse observationnelle » qui se focalise sur le phénomène de la cyber-religion dans sa globalité afin d'évaluer la portée de son influence et de ses effets par appel à l'observation en ligne et à des enquêtes fondées sur des entretiens avec les usagers et les webmasters. La deuxième catégorie de recherches est fondée sur des « examens philosophiques et théologiques » qui abordent une seule problématique (par exemple celle de l'espace sacré) comme une manière d'interpréter l'influence d'internet et de suggérer comment internet peut être utilisé pour reconnecter les individus aux idées ou croyances religieuses. La troisième catégorie, le "développement théorique », met en avant le besoin de créer des cadres paradigmatiques pour interpréter les données empiriques qui émergent des études de communication médiatisée par l'ordinateur. Enfin, l'« ethnographie sociale », qui étudie différentes cultures et communautés en ligne, se penche sur les problématiques liées aux pratiques de communication et à la communauté religieuse en ligne (Bratosin, Tudor et Coman, 2012). 


\section{Mihaela Alexandra TUDOR}

En France, les questions liées à la religion et aux (nouveaux) médias ont été plutôt explorées par la psychologie, la sociologie ou l'anthropologie. II est donc difficile d'affirmer qu'il existe une masse critique d'études et des recherches dans le domaine des sciences de la communication. Cependant, dernièrement, on pourrait remarquer un intérêt accru, qui a le potentiel de devenir systématique, à travers les études sur la communication du symbolique, du sacré et de la religion. Ces études sont valorisées aussi bien dans des colloques internationaux thématiques que dans des dossiers de revues scientifiques (Questions de communication, Essachess - Journal for Communication Studies, MEl, etc.) ou des réseaux de chercheurs (larsic, Relicom, etc.). Toutes ces contributions ont le mérite de reprendre les interrogations actuelles sur la question du religieux sans délimitation des frontières et celle des (nouveaux) médias qui intéressent les chercheurs à l'échelle internationale en valorisant la spécificité des approches de l'école française des sciences de la communication. Nous faisons surtout référence aux contributions françaises sur la médiation qui sont susceptibles d'apporter des arguments pour consolider l'idée récente des approches anglo-saxonnes sur les (nouveaux) médias et la religion. Des auteurs (Hoover, 2001; Carey, 2002 ; Hoover, 2006) suggèrent qu'il est problématique de considérer que les (nouveaux) médias et la religion sont des phénomènes distincts. En réalité, les (nouveaux) médias et la religion impliquent des processus similaires de médiation entre le sacré et le profane, entre le religieux et le séculaire (Hoover, 2001; Carey, 2002), pour lesquels il peut y avoir des similarités tel que l'occupation des mêmes espaces, même s'ils ne sont pas dans une relation d'harmonie.

\subsection{Concepts}

Face aux développements technologiques contemporains rapides dans un contexte de trans-médialité (Lister et al., 2008, p.11), les changements concernant la forme, la production, la distribution et les usages sont plus complexes que la distinction anciens et nouveaux médias le suggère puisqu'ils ont euxmêmes leur histoire et leurs précédents (Babou, 1998; Castells, 1999). Les nouveaux medias - les nouvelles formes des nouvelles technologies (networked technologies) - se placent au croisement de différents domaines d'étude.

tic\&société - 9 (1-2), 2015 
Multimédiatisation et événement religieux

Plusieurs disciplines s'en revendiquent la paternité et un nombre important de débats théoriques divisent les efforts des scientifiques à la recherche au moins d'une unité plurielle. Cela fait «qu'on débat aujourd'hui dans le désordre, des "médias sociaux", du "printemps arabe", d'internet, des blogs, des SMS, de Twitter et de leurs avatars... 》 (Palmer et Nicey, 2012, p.107).

L'évolution rapide $\mathrm{du}$ web et son impact sur les comportements, les opinions, les choix, les croyances, etc. des millions de gens sont plus que significatifs. Aujourd'hui, une connexion réseau et n'importe quel appareil suffisent à créer du contenu riche et interactif. II est incontestable que l'expansion rapide des nouveaux médias et des médias sociaux en particulier n'a pas laissé indifférent le paysage médiatique traditionnel (Serafeim, 2012; Becker et Vlad, 2012).

Dans ce contexte, deux approches sur les nouveaux médias nous semblent articuler un cadre conceptuel opérationnel pour répondre à notre problématique: celle de Heidi Campbell (2010) et celle de Lev Manovich (2001).

Les innovations technologiques dans les médias ont remis en cause les formes de technologie et leurs modes d'utilisation établis auparavant, en créant de nouvelles formes considérées à l'échelle de l'histoire de toutes les technologies comme une sorte de nouveauté réelle. Selon Heidi Campbell (2001), les nouveaux médias représentent la génération des médias qui se détachent dans un paysage contemporain et qui offrent de nouvelles opportunités pour l'interaction sociale, le partage de l'information et la communication médiatisée. Une négociation se produit lorsqu'une nouvelle forme de technologie émerge et est introduite par un média religieux (Bratosin, Tudor et Coman, 2012) - c'est à cette négociation que le présent article s'intéresse et plus précisément aux modèles qu'elle pourrait générer.

L'approche de Lev Manovich (2001) a le mérite de mettre en exergue ce qui est vraiment nouveau dans ces formes de médias. Manovich tente d'identifier des théorisations « aptes à désigner ce qui est propre aux nouveaux moyens de communication. II dénonce l'imprécision de notions très répandues, telle que celles de "multimedia" et de "digital" » (De lulio, 2003, p.1). Tout d'abord, Manovich définit les nouveaux médias en fonction de cinq caractéristiques qui mettent en évidence en quoi les technologies numériques ne sont pas 


\section{Mihaela Alexandra TUDOR}

différentes par rapport à celles qui les ont précédées. II s'agit de la représentation numérique, la modularité, l'automation, la variabilité et la transcodification (Manovich, 2001).

La représentation numérique se réfère à la numérisation ; les objets réalisés et véhiculés par les nouveaux médias sont codés numériquement, sont décrits mathématiquement et soumis à la manipulation algorithmique. La modularité indique que les nouveaux médias ont une structure modulaire, composée d'éléments discrets et indépendants, qui peuvent être manipulés séparément. La modularité permet l'automation de nombreuses opérations impliquées dans le processus de création de médias, la manipulation et l'accès sans que l'usager intervienne directement puisque certaines causalités sont programmées dans ces nouveaux médias. La variabilité suppose que les produits des nouveaux médias ont des versions potentiellement infinies. La codification des éléments médias signifie que les informations structurales sont stockées dans un format de base de données. Ceci permet à différentes interfaces d'interagir avec ces données; plusieurs formes peuvent être créées à partir des mêmes données. Ceci permet également aux programmes informatiques le recueil de données sur l'usager afin de personnaliser automatiquement la composition de la forme des nouveaux médias. La dernière caractéristique des nouveaux médias, la transcodification, exprime le fait que les technologies des nouveaux médias ont en elles-mêmes la capacité d'être facilement traduites d'un format vers un autre.

Ces approches nous aident à identifier trois paradoxes dominants dans la compréhension des spécificités des nouveaux médias.

Le premier paradoxe consiste à trancher, même après trente ans, entre ce qui est vraiment nouveau et ce qui ne l'est qu'en apparence, car il existe depuis longtemps des médias qui échappent à la régularisation facile, des genres ou des intertextes hybrides (Babou, 1998 ; Castells, 1999 ; De Vries et Weber, 2001 ; Manovich, 2001 ; Lister et al., 2008 ; Campbell, 2010).

Le deuxième paradoxe est né de la dynamique de la confrontation entre les anciens et nouveaux médias en termes de présupposés. Nous sommes confrontés à un ensemble continu d'expériences technologiques et d'initiatives 
Multimédiatisation et événement religieux

entrepreneuriales, d'un côté, et de l'autre côté, à un ensemble complexe d'interactions entre les nouvelles possibilités technologiques et les formes de médias établis avec des enjeux culturels profonds; les présupposés fondateurs dominants des nouveaux médias semblent être techniques, or leur dimension symbolique nous oblige à considérer également les présupposés symboliques, plus précisément les rapports avec les grands facteurs structurants des anciens médias : systèmes de valeurs et d'habitudes, idéologie, etc. ce qui change la donne (Babou, 1998 ; Castells, 1999 ; Lister et al., 2008).

Cela nous amène au troisième paradoxe: la notion de nouveaux médias semble être utilisée sans contestation, alors qu'il s'agit plutôt d'une notion porte-manteau, avec des enjeux symboliques importants pour au moins trois raisons. Premièrement, cette terminologie témoigne du fait que, pour l'instant, elle est la seule à désigner la pluralité et complexité des tendances historiques actuelles en matière de développement technologique. Deuxièmement, le mot « nouveau » a un fort pouvoir idéologique et utopique car il est connoté positivement, associé à l'avant-garde, à l'idée de progrès, rassemblant ainsi tout un héritage du modernisme articulé sur l'idéologie postmoderne de l'innovation (terme remplaçant aujourd'hui celui de progrès). Troisièmement, l'expression est un mot «bateau » car elle évite de réduire les médias à des termes trop techniques et spécialisés (tels médias digitaux ou électroniques).

Nous pouvons faire l'hypothèse que, finalement, les nouveaux médias sont une abstraction qui laisse la place à la variété, à tout ce qui est lié de manière directe ou indirecte aux technologies (pour illustration, ils englobent la communication médiatisée par l'ordinateur, les nouvelles manières de distribuer et consommer les médias textes, une pléthore de transformations et de dislocations des médias classiques - la photographie, l'animation, la télévision, le cinéma -, des réalités virtuelles, etc.).

Dès lors, nous explorerons le design du dispositif média et le modèle d'engagement du média adventiste Hope Channel Romania - Speranta TV avec la variété de nouvelles formes de médias numériques et avec internet, ce laboratoire où il paraît que «toutes les expérimentations sont légitimes » (Bratosin, 2014, p.15), par l'étude de cas de la campagne d'évangélisation l' " Horizon de l'espérance ». Les discussions du matériel empirique s'appuieront sur l'appareillage théorique 
et conceptuel présenté ci-dessus.

\section{Comment les médias religieux s'engagent- ils avec les nouveaux médias? La campagne d'évangélisation I' " Horizon de l'espérance »}

\subsection{Hope Channel Romania : engagements avec les médias traditionnels et les nouveaux médias}

Avant de présenter le matériel empirique, il est nécessaire de placer la campagne d'évangélisation de Speranta TV dans le contexte plus large du réseau mondial Hope Channel. Hope Channel Romania - Speranța TV fait partie du réseau international de télévision néo-protestante adventiste Hope Channel, né en 2003 et comprenant aujourd'hui 23 chaînes qui transmettent en 12 langues par câble, satellite et sur internet en Amérique du Nord et du Sud, en Europe, en Asie, en Afrique, en Australie et dans les îles du Pacifique (Dumitrascu, 2014 a).

La grille des programmes TV de la chaîne roumaine comprend des productions propres en roumain et en hongrois $(80 \%)$ et des productions réalisées par les chaînes d'autres pays du réseau, sous-titrées en roumain (20\%). La grille comporte des émissions sur des thèmes sociaux, culturels, en mettant l'accent sur les questions d'éducation et de santé, de famille et de bien-être. À titre d'exemple, en 2012, l'émission produite et diffusée par Speranta TV intitulée "Le miracle de l'organisme humain " a remporté un prix décerné par l'Association des professionnels de télévision de Roumanie (Idem).

Dans un pays comportant une écrasante majorité de chrétiens orthodoxes, Speranta TV est la chaîne de télévision néo-protestante la plus importante de Roumanie ${ }^{2}$. Son public est divers: adventistes du septième jour, baptistes, pentecôtistes et évangéliques, catholiques et orthodoxes. En 2014, le Conseil national de l'audiovisuel a inclus Speranta TV

\footnotetext{
${ }^{2}$ II existe encore deux chaînes de télévision néo-protestantes : Alpha et Omega et Credo TV.
}

tic\&société - 9 (1-2), 2015 
Multimédiatisation et événement religieux

dans la liste des télévisions must carry ${ }^{3}$, ce qui signifie encore une plus grande visibilité auprès des téléspectateurs (Ibidem).

Speranta TV utilise à la fois les supports et médias traditionnels et les nouveaux supports et médias, étant la première chaîne de télévision de Roumanie qui a commencé à transmettre en direct 24 heures par jour sur Facebook à partir de 2009. En 2014, elle devient la première télévision de Roumanie connectée au lecteur de diffusion Roku. Au-delà de son public habituel, Speranta TV a un public formé des téléspectateurs de diverses confessions de la diaspora roumaine qui regardent la version web de la télévision sur internet ou sur la page Facebook. Parmi les téléspectateurs diasporiques qui regardent la chaîne sur internet, les plus connectés aux programmes de Speranta TV se trouvent principalement en Espagne, aux États-Unis, en Allemagne et en Italie. II s'agit, en effet, des pays qui comptent le plus grand nombre d'immigrés roumains. Selon les statistiques des indicateurs internet, il y a une distribution équilibrée par tranche d'âge du public qui regarde la télévision en ligne. Un tiers du public se trouve dans la tranche 25-34 ans (Dumitrascu, 2014 a).

\subsection{Matériel empirique et méthodologie}

L'Institut théologique adventiste de Bucarest, université habilitée de l'Église adventiste roumaine, a accueilli entre le 6 et le 14 décembre 2013 une série de conférences bibliques intitulée l'« Horizon de l'espérance ». La télévision Speranta TV a diffusé en direct cet évènement par câble, satellite et internet en même temps que la radio Voix de l'Espérance ${ }^{4}$, qui avait repris le programme d'évangélisation transmis à la télévision. Les dix conférences données en anglais ont été traduites en

\footnotetext{
${ }^{3}$ Désigne l'obligation pour un distributeur de services par câble ou par satellite de reprendre une chaîne de télévision.

${ }^{4}$ La radio Voix de l'espérance est la radio officielle de l'Église adventiste du septième jour de Roumanie. Elle fait partie du réseau mondial Adventist World Radio, fondé en 1971, qui transmet dans le monde entier dans plus de 70 langues. En Roumanie, Voix de l'espérance fonctionne depuis 1992. Actuellement, il y a un réseau de 45 stations locales propres qui transmettent 24 heures chaque jour et cinq stations locales propres qui transmettent de deux jusqu'à 18 heures par jour. La radio Voix de l'espérance est accessible par satellite et internet (http://www.rvs.ro/despre-noi.html, dernière consultation le 24 août 2015).
} 


\section{Mihaela Alexandra TUDOR}

quatre langues - roumain, hongrois, russe et ukrainien - et ont été simultanément diffusées en Roumanie, ainsi qu'en Ukraine et en République de Moldavie via le câble, le satellite et internet. La campagne d'évangélisation ${ }^{5}$ a eu comme protagoniste le pasteur américain Douglas (Doug) Batchelor, écrivain et président de l'organisation médiatique américaine Amazing Facts. S'appuyant sur ses propres expériences, Batchelor a présenté des épisodes significatifs de la vie des personnages bibliques dans le contexte du vingt-et-unième siècle. Chaque conférence a été suivie d'un débat télévisé en direct avec des participants du monde académique, laïc et religieux, et des représentants des organisations ecclésiales de diverses confessions.

Le matériel empirique sur lequel se fonde cette recherche repose sur les données recueillies lors de l'observation non participante, les données statistiques des réseaux sociaux et internet (les sites web de Speranta TV, sperantalaorizont.ro, adventube.ro et hopetv.org, Google Analytics, Youtube et la page Facebook de Speranta TV), les rapports officiels et documents internes mis à disposition par la productrice du programme et directrice de marketing de la chaîne.

Quant au choix méthodologique, il s'agit d'une recherche exploratoire qui s'appuie sur une méthode composite, articulée sur le principe des feuilles d'oignon (i.e. couche par couche), dans le but de rechercher un nombre suffisant de données afin de comprendre le contexte de l'intérieur sans y participer et de pouvoir isoler le modèle de communication créé par ce média

\footnotetext{
${ }^{5}$ La multiplication des discours institutionnels, scientifiques, politiques, médiatiques, religieux, etc. (notamment sur la Toile et dans le paysage multimédiatique complexe très sensible à la façon de transmettre et communiquer le message religieux), la pléthore d'acteurs qui s'emparent du sujet de l'évangélisation et ses déclinaisons ont entraîné, au-delà d'une simple banalisation et de toute autre interprétation plus ou moins doctrinaire ou idéologique, l'apparition d'une "culture de la suspicion » qui la perçoit et la qualifie soit de prosélytisme, soit de publicité cachée, soit de marketing, soit de propagande... II devient alors important de préciser le sens de l'évangélisation dans le contexte créé par notre terrain. Le projet l' " Horizon de l'espérance » a été conçu comme un ensemble d'actions de l'Église adventiste roumaine en concertation avec l'Église adventiste mondiale à forte dimension missionnaire qui a visé à présenter l'Évangile à la fois aux non-croyants et aux croyants, à véhiculer, diffuser, propager la foi chrétienne et à sensibiliser le grand public à l'Évangile à travers tous les médias de masse à disposition, avec un accent particulier porté sur l'utilisation des nouvelles découvertes technologiques (smartphones, tablettes, Twitter, Facebook, etc.) (Dumitrascu, 2014 a).
}

tic\&société - 9 (1-2), 2015 
Multimédiatisation et événement religieux

religieux face aux défis des NTIC dans le cas concret d'une campagne d'évangélisation. Cette méthode mobilise :

1) L'observation non participante ${ }^{6}$ qui est beaucoup plus appropriée pour le recueillement d'informations dans le cas des phénomènes de médias de masse car elle donne la possibilité de se confronter à la réalité sans y intervenir (Winkin, 1997 ; Angrosino et Mays de Pérez, 2000 ; Berger, 2000 ; Becker, 2003 ; Emerson, 2003).

Présente sur le plateau de télévision lors de la préparation du direct et de la transmission en direct des conférences bibliques, nous avons mené un travail d'observation qui s'est principalement intéressé à l'organisation du dispositif multimédia mis en place pour cette évangélisation, à son fonctionnement et au modèle médiatique qu'il avait généré. Nous avons négocié une position de simple observateur, durant la période du 6 décembre jusqu'au 14 décembre 2013, sans aucune implication dans le projet, sans aucun rôle fonctionnel dans le groupe de travail. La négociation de cette posture a été facilitée par notre qualité de collaborateur permanent dans un autre projet, une émission de télévision de médiatisation de la recherche scientifique, sans objectif spirituel ou religieux. Nous avons pu observer les activités de la productrice et directrice de marketing, du directeur de la chaîne, des modérateurs et réalisateurs, de l'équipe technique sans que nous soyons perçue comme un élément intrusif, la préservation de la qualité des observations faisant partie de nos objectifs. Les informations recueillies lors de l'observation ont été corroborées avec les autres données à notre disposition et restituées dans la discussion du matériel empirique.

2) L'analyse des documents suivants: indicateurs statistiques des réseaux sociaux et internet mis à disposition par l'équipe de direction de la chaîne avant et après l'événement, rapports officiels publiés ainsi que les documents internes de travail.

\subsection{Présentation des échantillons et résultats. Discussion}

Dans ce qui suit, nous présenterons quelques échantillons du matériel empirique qui nous serviront à introduire d'une part

\footnotetext{
${ }^{6}$ Même si « l'observation est naturellement participante » (Yves Winkin, 1997).
} 


\section{Mihaela Alexandra TUDOR}

les différentes façons par lesquelles les médias traditionnels ont été mis en relation avec la Toile et d'autre part le fond empirique pour les discussions.

Un premier échantillon comprend les données statistiques correspondant aux métriques d'évaluation du dispositif de communication multimédia mis en place pour le programme l' « Horizon de l'espérance ». ${ }^{7}$

Suite aux deux campagnes simultanées hors ligne et en ligne menées par Speranta TV du 17 novembre au 16 décembre 2013 (un mois), le site web de Speranta TV a enregistré 44542 visiteurs uniques (figure 1) dont 17,60\% de nouveaux visiteurs ; un chiffre important compte tenu que le site enregistrait, avant cette période, 60000 visiteurs depuis sa création en 2007. Les visiteurs sont restés sur le site en moyenne 4 minutes et 19 secondes. Le nombre de pages consultées a été de 2,62 par visite. Ces paramètres statistiques s'inscrivent dans une courbe plutôt positive si l'on considère les chiffres portant sur les indicateurs tels le temps moyen passé sur un page web (30 à 60 secondes), le temps moyen passé sur un site ( 2 minutes), le nombre moyen de pages consultées sur un site ( 2 ; cf. Canivet, 2014). Le taux de rebond (bounce rate) est intéressant. II est situé à $54,90 \%$. Si on le corrèle avec le temps passé sur le site, on remarque un résultat positif de l'intérêt et de la satisfaction montrés par les visiteurs et cela dans le contexte où, dans cette période, la première page du site a été alimentée en continu avec des vidéos et des informations sur la série de conférences bibliques. La publication des horaires de l'évènement a servi d'aiguillage et a été «à la une » de l' " Horizon de l'espérance », sans qu'il soit nécessaire de rechercher l'information sur l'ensemble du site internet.

\footnotetext{
${ }^{7}$ Les données ont été mises à notre disposition grâce à l'amabilité du Centre Média Adventiste Speranta (par la productrice du programme qui est également la directrice marketing de Speranta TV).
} 
Multimédiatisation et événement religieux

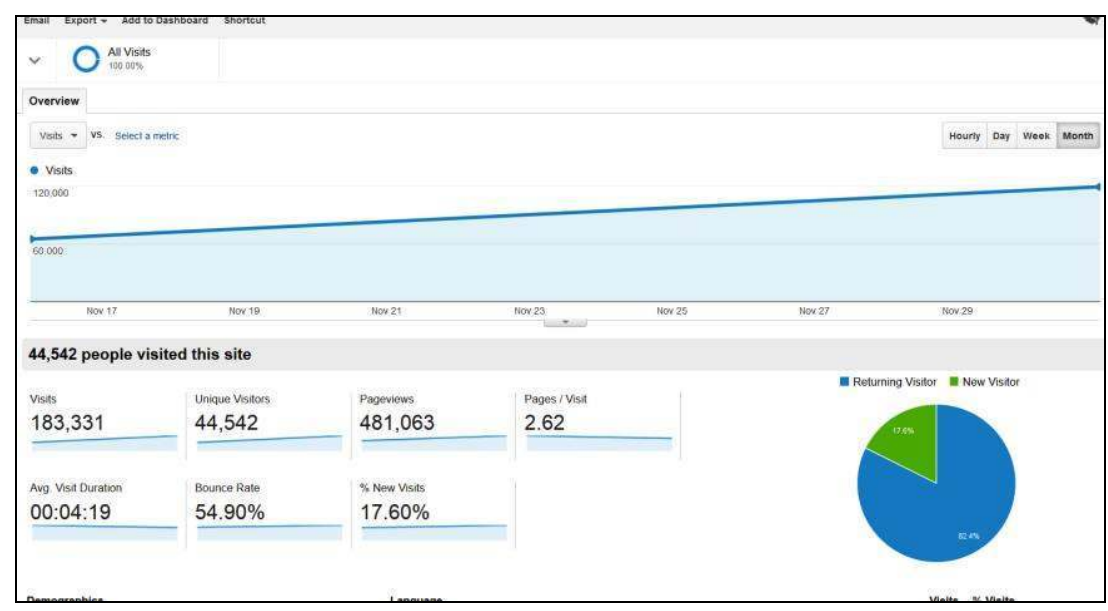

Figure 1. Visites sur le site Speranta TV du 17 novembre au 17 décembre 2013.

En ce qui concerne les visites du site Speranta $T V$, les résultats (figures $2 \mathrm{a}, 2 \mathrm{~b}$ et 3 ) nous montrent que les conférences transmises au premier et au dernier jour du programme l' "Horizon de l'espérance " ont été les plus consultées. Le nombre de personnes qui ont visionné les conférences sur le site web a été de 13119 dont $26,56 \%$ de nouvelles visites le 6 décembre et de 15580 le 14 décembre, s'agissant d'une croissance de 2461 spectateurs-internautes le dernier jour par rapport au jour du début de la série (Dumitrascu, 2014 b).

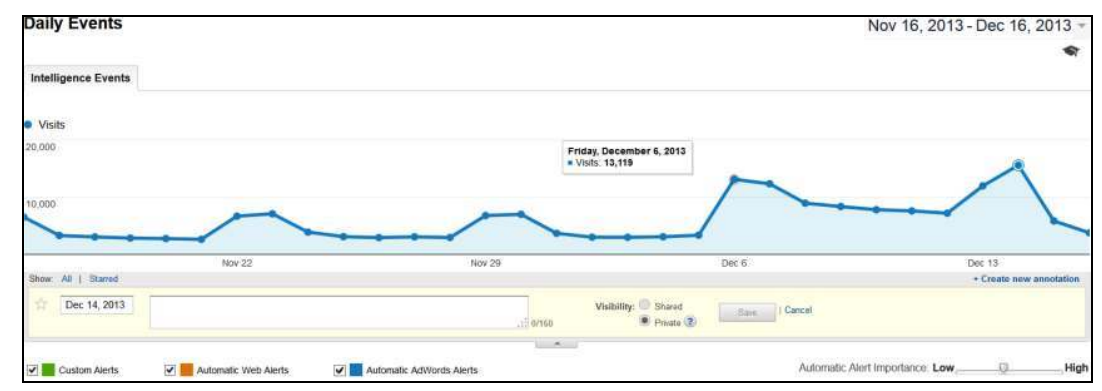

Figure 2a. Visites du site Speranta TV le 6 décembre 2013. 
Mihaela Alexandra TUDOR

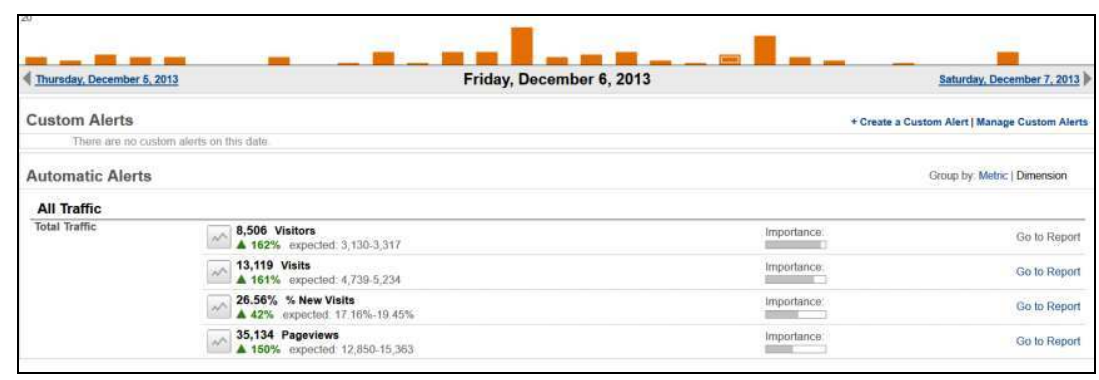

Figure 2b. Visites du site Speranta TV le 6 décembre 2013 (détail).

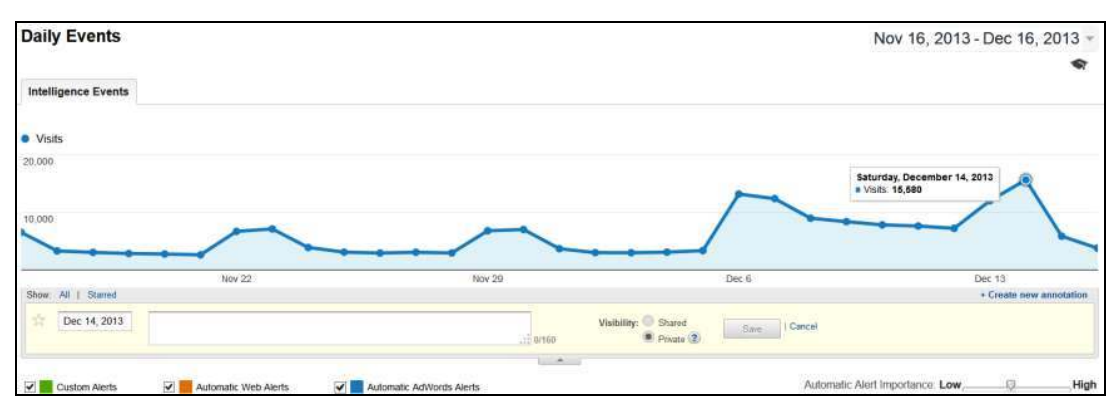

Figure 3. Visites du site Speranta TV le 14 décembre 2013.

Durant la période de transmission en direct du programme, plus de 150000 visites cumulées des lecteurs multimédia des sites web sperantatv.ro, sperantalaorizont.ro, adventube.ro, et hopetv.org ont été faites conformément aux statistiques Google Analytics (Dumitrascu, 2014 b). Le lecteur multimédia de transmission en direct du site sperantatv.ro a enregistré 84960 lectures. Le temps moyen de lecture a été de 22 minutes. 13\% des internautes (captivated) ont passé moins de 30 minutes, mais plus de 10 minutes et $25 \%$ (fans) ont passé moins d'une heure, mais plus de 30 minutes (figure 4). 
Multimédiatisation et événement religieux

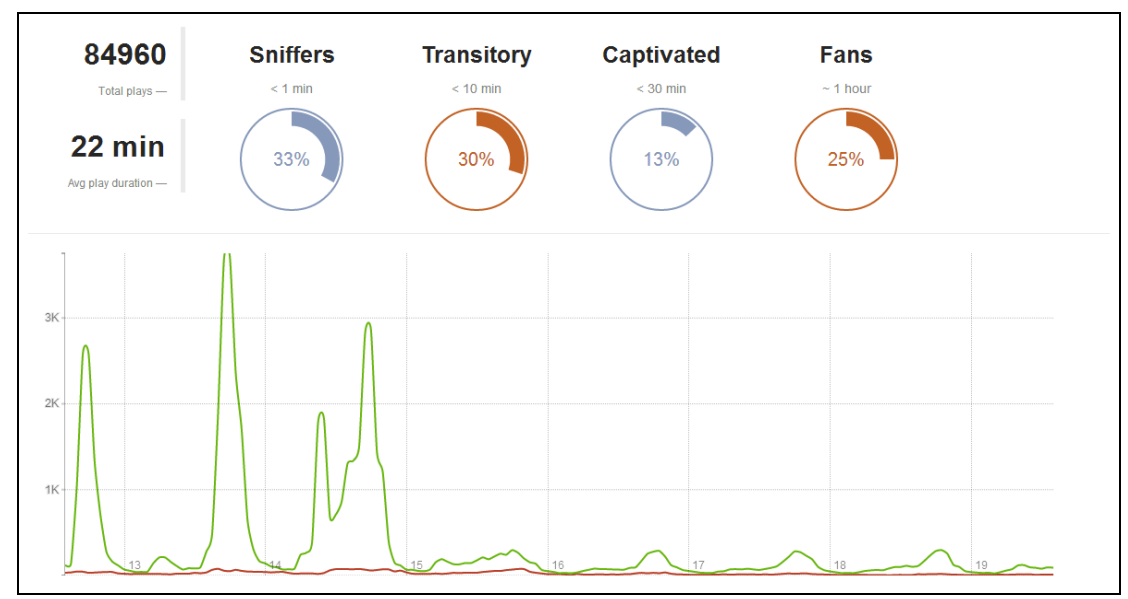

Figure 4. Statistiques du visionnage en direct de l'Horizon de l'espérance par le lecteur multimédia du site sperantatv.ro.

Un total de 193000 lectures du lecteur multimédia des conférences enregistrées ont été faites sur le site sperantatv.ro par les internautes. Le temps moyen de lecture a été de 22 minutes. $13 \%$ des internautes (captivated) ont passé moins de 30 minutes, mais plus de 10 minutes et $25 \%$ (fans) ont passé moins d'une heure, mais plus de 30 minutes (figure 5).

Les conférences ont été disponibles en ligne à visionner et/ou à télécharger. Plus de 8800 téléchargements ont été effectués : environ 5400 en traduction roumaine, 1900 en traduction hongroise, 1400 en traduction russe, et 170 en traduction ukrainienne (Dumitrascu, 2014 b). 
Mihaela Alexandra TUDOR

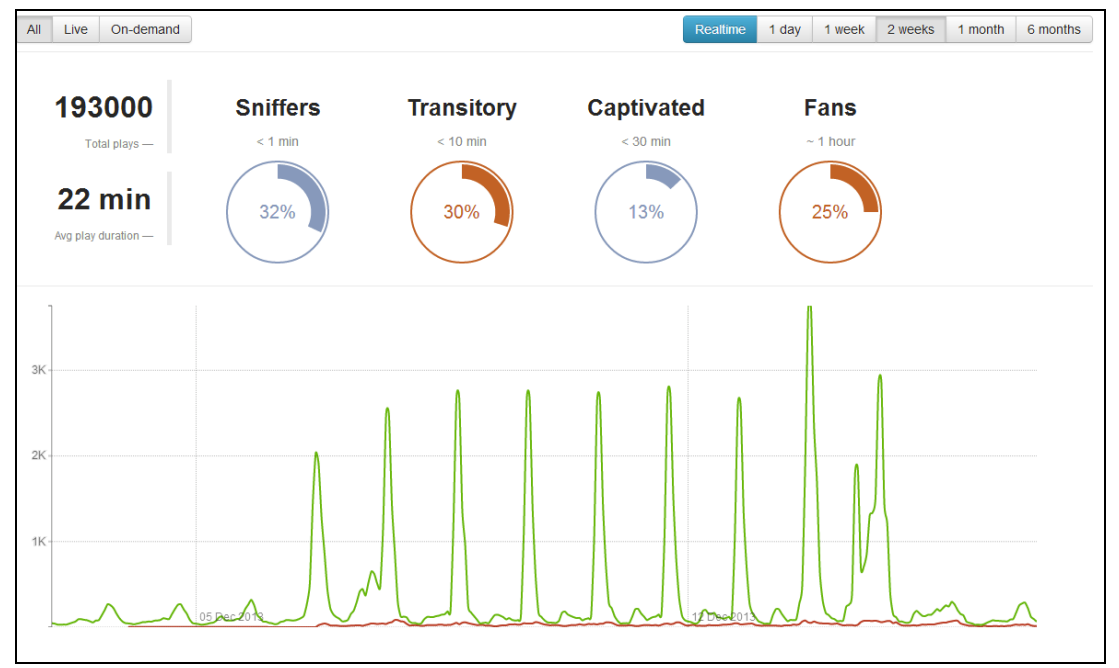

Figure 5. Statistiques du visionnage et des enregistrements l'Horizon de l'espérance dans le lecteur multimédia du site sperantatv.ro.

La page Facebook Speranta TV met en lumière de manière plus claire le degré d'intérêt suscité par la série des conférences bibliques. La portée sur les fans, indicateur-clé qui relie le contenu, l'audience et la qualité de celle-ci, a évolué de manière spectaculaire. Si depuis 2009, l'année de création de la page Facebook, Speranta TV avait seulement 5700 fans, en deux semaines (celle d'avant et celle de la transmission en direct des conférences), 10000 fans se sont ajoutés au chiffre initial. $58 \%$ des fans représentent des femmes, $42 \%$ d'entre eux représentent des hommes (Dumitrascu, 2014 b). Si l'on considère les pourcentages des fans parmi les femmes par tranche d'âge, on note la situation suivante : 4\% d'entre elles ont entre 13 et 17 ans, $15 \%$ ont entre 18 et 24 ans, $15 \%$ ont entre 25 et 34 ans, $12 \%$ ont entre 35 et 44 ans, $7 \%$ ont entre 45 et 54 ans, $4 \%$ ont entre 55 et 64 ans et $1 \%$ ont 64 ans et plus. Les pourcentages des fans parmi les hommes par tranches d'âge sont : $3 \%$ ont entre 13 et 17 ans, $12 \%$ ont entre 18 et 24 ans, $11 \%$ ont entre 25 et 34 ans, $9 \%$ ont entre 35 et 44 ans, $4 \%$ ont entre 45 et 54 ans, $2 \%$ ont entre 55 et 64 ans et $1 \%$ ont 64 ans ou plus (cf. rapports internes du Centre Média Adventiste Speranta mis à disposition en mai 2014).

Les portées organiques et payées, c'est-à-dire le nombre total de personnes ayant vu les posts par le biais d'une

tic\&société - 9 (1-2), 2015 
Multimédiatisation et événement religieux

distribution non payante et par une publicité, ont augmenté simultanément. Â titre d'exemple, si la moyenne de la portée organique au mois de novembre était de 2055 , la moyenne dans la période de transmission des conférences augmente de manière évidente à 6879 (figure 6).

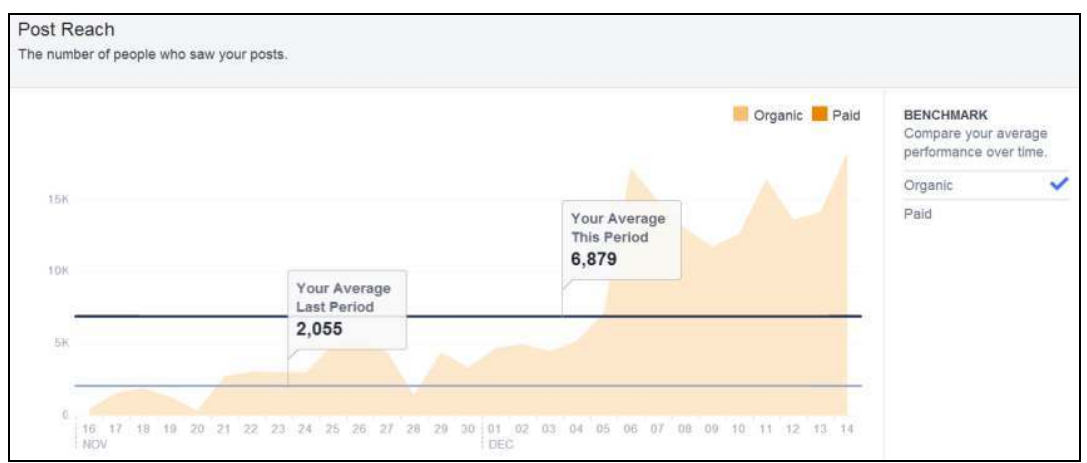

Figure 6. Portée organique novembre-décembre 2013.

Après la portée sur les fans, l'engagement des posts défini par Facebook comme «le nombre de personnes ayant cliqué n'importe où sur votre publication » (<https://frfr.facebook.com/help/336143376466063/> \& ), représente l'indicateur le plus important de ce réseau. Plus précisément, il se réfère à un "j'aime », un commentaire ou un partage, aux personnes qui ont vu une vidéo, qui ont cliqué sur un lien, sur une photo, sur le nom d'une personne mentionnée, qui ont aimé un commentaire, ou bien qui ont donné un feedback négatif. Par exemple, la vidéo de promotion de l' "Horizon de l'espérance " ayant comme protagoniste le pasteur Doug Batchelor a recueilli 1286 engagements des posts. Effectivement, 1286 des internautes ont agi sur ce contenu, ont passé du temps à le lire. L'audience de la vidéo a été de 63854 .

Un autre contenu publié sur la page Facebook qui nous offre des indicateurs statistiques interessants est la photo des présentateurs de la série des conférences. II s'agit, plus précisément, de la photo sur laquelle ils étaient habillés en costume national et accompagnés d'un enfant issu du public. La portée organique touche 5360 utilisateurs, le total cumulé des j'aime, commentaires et partages étant de 480 et le total

${ }^{8}$ Dernière consultation le 23 août 2014. 


\section{Mihaela Alexandra TUDOR}

des post clicks de 1954 dont 1048 photo views.

Un deuxième échantillon comprend les données statistiques portant sur l'accès à internet et l'utilisation des médias sociaux en Roumanie, données censées offrir le panorama de la couverture du programme l' "Horizon de l'espérance » au travers des nouveaux médias. En Roumanie, $53 \%$ des ménages sont équipés d'internet à domicile dont $73 \%$ dans les zones urbaines. $65 \%$ d'entre eux utilisent internet chaque jour. $95 \%$ des ménages équipés se connectent via les connexions au débit fixe, $62 \%$ via le portable et $48 \%$ via l'ordinateur portable (cf. dernier rapport de l'Institut national de statistique de Roumanie, 2013). En ce qui concerne l'utilisation des médias sociaux, il y a 7200000 usagers de Facebook, c'est-àdire $36 \%$ de la population du pays, $75 \%$ d'utilisateurs d'internet possédant un compte sur Facebook dont 13\% ont entre 25 et 49 ans. 105000 roumains utilisent Twitter dont 30\% sont des utilisateurs quotidiens. 714000 ont des comptes sur Youtube et 1290000 sur Linkedln (cf. figure 7, facebrands.ro). 
Multimédiatisation et événement religieux

\begin{tabular}{|c|c|c|}
\hline \multicolumn{3}{|c|}{ Social Media in Romania (Feb 2014) } \\
\hline facebook & $\begin{array}{l}7,200,000 \\
\text { user accounts } \\
\text { FacB }\end{array}$ & NA \\
\hline Linkedin & 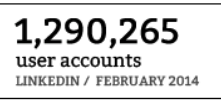 & NA \\
\hline trilullilu & NA & 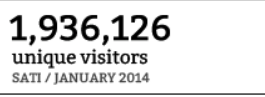 \\
\hline loil $P$ & $\begin{array}{l}760,164 \\
\text { user accounts } \\
\text { trouro }\end{array}$ & 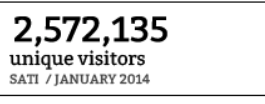 \\
\hline foursquare & $\begin{array}{l}\text { 90,350 } \\
\text { user accounts } \\
\text { ZEUST/ FERRARY 2014 }\end{array}$ & NA \\
\hline Ewibter & 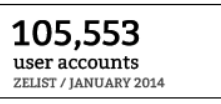 & $\begin{array}{l}17,345 \\
\text { active users } \\
\text { zEUS / ANVAR 2014 } \\
\end{array}$ \\
\hline BLOGGING & 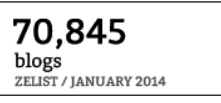 & $\begin{array}{l}12,069 \\
\text { active blogs } \\
\text { zLUS / IAvUAR 2014 }\end{array}$ \\
\hline Instagram & $\begin{array}{l}75,181 \\
\text { user accounts } \\
\text { zEUS / IANUAR 2014 }\end{array}$ & $\begin{array}{l}49,036 \\
\text { active users } \\
\text { zEUST/ANUARY 2014 }\end{array}$ \\
\hline YouTube & 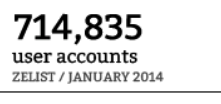 & 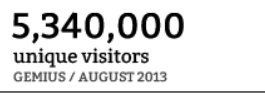 \\
\hline & NA & 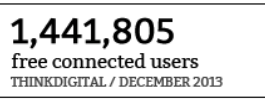 \\
\hline $\begin{array}{l}\text { NA= Non Avaliable } \\
\text { See each sourc below the figures }\end{array}$ & & $\begin{array}{l}\text { Cristian Manafu/ Febrary } 2014 \\
\text { wwwsocialmediatrainingroro }\end{array}$ \\
\hline
\end{tabular}

Figure 7. Médias sociaux en Roumanie (cf. facebrands.ro).

Ces données doivent être mises en rapport avec le troisième échantillon, les statistiques portant sur la structure religieuse de la population roumaine et les performances média de la chaîne de télévision de la confession majoritaire. La Roumanie est un pays majoritairement orthodoxe, $86,5 \%$ des roumains se déclarant chrétiens orthodoxes. $4,6 \%$ de la population est de confession romano-catholique, $3,2 \%$ reformée, $1,9 \%$ pentecôtiste, $0,8 \%$ gréco-catholique, $0,6 \%$ baptiste et $0,5 \%$ adventiste du septième jour conformément aux résultats communiqués par l'Institut national de statistique de Roumanie (INS) suite à la dernière campagne de recensement qui a eu lieu en 2011 (INS, juillet 2013). Le reste des personnes recensées se sont déclarées d'autres religions ou athées. II s'agit, donc, d'une population qui s'affirme majoritairement croyante dont un pourcentage très élevé de croyants se déclarent chrétiens orthodoxes, l'orthodoxie étant un très fort marqueur d'identité nationale. 


\section{Mihaela Alexandra TUDOR}

Historiquement le, paysage religieux roumain est dominé par le monopole de l'Église orthodoxe (Tanase, 2008; Chirila, 2014), avec une tolérance pour « la différenciation ethnique des expressions religieuses » (Tanase, 2008, p.83) et la présence des nouveaux mouvements religieux (NMR), notamment néoprotestants. Pourtant, dans la période communiste, l'État totalitaire avait imposé une politique de diminution du rôle du religieux et des institutions religieuses dans la vie politique, sociale et même privée, et mis en place une régulation étatique des cultes religieux par un système de cultes reconnus, strictement surveillés, ce qui empêchait ce monopole. L'effet fut inverse, l'Église orthodoxe étant " l'institution de base de la mémoire culturelle et de l'opposition » (Tomka apud Tanase, 2008, p.81). Après la chute du communisme, en 1989, l'Eglise orthodoxe garde cette primauté, mais les changements politiques et le cheminement de la Roumanie vers la démocratie permettent aux NMR de gagner du terrain non seulement dans l'augmentation du nombre des fidèles, mais aussi dans la disponibilité de réponse par l'action aux évènements et actions organisés par ces mouvements.

L'ouverture des frontières en 1989 a coïncidé d'une part avec la libre circulation et d'autre part avec le droit au travail à l'étranger de la population. Simultanément, un fort courant missionnaire et d'évangélisation sur un marché religieux libre et concurrentiel se créée en contribuant à la diminution de l'impact des discours et messages de dénigrement des NMR véhiculés par les médias orthodoxes sur la population ${ }^{9}$. Dans ce contexte, ces discours dans les médias orthodoxes ne sont plus prisés: la diaspora s'agrandit et les missionnaires venus d'Occident les ont transformés en discours galvaudés.

Les NMR, historiquement connectés à tous les développements technologiques (l'Eglise orthodoxe les intègre plutôt formellement, sans toucher réellement ce public vaste qui est orthodoxe, mais qui ne fréquente pas les médias orthodoxes $\left.{ }^{10}\right)$, ont gagné plus d'attention de la part de cette

\footnotetext{
${ }^{9}$ Les NMR ont été toujours qualifiés de sectes avant la chute du communisme et même après.

${ }^{10}$ L'Eglise Orthodoxe roumaine est propriétaire du centre média Basilica comprenant le journal Lumina, la chaîne de télévision Trinitas TV et la radio Trinitas. Malgré la disponibilité par câble, satellite et sur internet de la chaîne de télévision Trinitas, sa faible audience reste un phénomène à analyser. Entre le 1 janvier et le 31 décembre 2013, Trinitas TV enregistrait une audience de 0,073 (cf. document interne mis à
}

tic\&société - 9 (1-2), 2015 
Multimédiatisation et événement religieux

population majoritairement orthodoxe, non forcément en terme d'adhésion, mais en terme d'assiduité pour le suivi de leurs activités, de leurs actions, de leurs projets, etc. Ce suivi est fortement visible dans l'audience des médias religieux néoprotestants et, surtout, dans le cas des événements organisés au niveau du réseau mondial. Manifestement, dans le paysage médiatique religieux roumain, le seul cas de figure qui réunit toutes ces conditions est celui des médias adventistes Hope Channel.

Dans ce cadre, une hypothèse peut être faite concernant l'événement l' "Horizon de l'espérance »: le dispositif multimédia hybridé créé pour ce programme a facilité une large circulation du contenu, un enrichissement effectif sur plusieurs supports plus disponibles (anciens supports comme le papier, la télévision et la radio et les nouveaux supports comme internet, les mobiles, les plateformes, les réseaux sociaux, les applications), une diversification créative des activités médiatiques, une valorisation des produits sur plusieurs activités. Dans le cas de cette campagne d'évangélisation mise en place par Speranta TV, nous considérons qu'il ne s'agit pas d'un «transfert d'autorité » des anciens médias vers les nouveaux médias, mais plutôt d'un engagement dans la logique de la convergence (Jenkins, 2006). La convergence repose non pas sur le paradigme du digital - tout évolue vers un seul dispositif intégrateur entre les formes classiques et nouvelles des médias (Jenkins, 2006; Ruellan, 2012), mais sur le paradigme du mutualisme - les deux tirant profit de cette interaction (Manovich, 2001). Ce dispositif multimédia mis en place pour la transmission de la parole biblique est l'expression d'un engagement de mise en synergie, de partage des ressources et de leurs interactions, et non d'un engagement de type full integrated system, d'un dispositif universel central à télécommande universelle. À ce stade, le cas d'étude nous permet de faire encore une hypothèse : les fonctions et statuts du média religieux ne sont pas remplacés par les nouvelles technologies, mais déplacés vers les nouvelles technologies.

disposition en 2014 par le Conseil national de l'audiovisuel de Roumanie aux télévisions must carry parmi lesquelles Speranta TV, Alpha et Omega TV, etc.) pour une population de presque $87 \%$ de chrétiens orthodoxes. Qui plus est, sa page Facebook créée en 2011 compte à ce jour seulement 2411 fans (cf. https://roro.facebook.com/TrinitasTV, dernière consultation le 23 juin 2014), un chiffre dérisoire rapporté aux nombres de croyants déclarés orthodoxes. 


\section{Mihaela Alexandra TUDOR}

II s'agit d'intégrer les NTIC dans le sens de " l'acculturation à des activités nouvelles » (Ruellan, 2012, p.1) qui peuvent être développées simultanément et parallèlement au média religieux classique. II ne s'agit donc pas d'un engagement qui favorise l'exclusion des médias traditionnels ou la domination sur les médias traditionnels, mais d'une synergie par réinvention, recalibrement et repositionnement de ceux-ci face aux défis des NTIC.

Nous allons maintenant prendre en considération le quatrième échantillon, les indicateurs statistiques d'audience. Un total de 328 églises a regardé le programme par satellite en Roumanie (Dumitrascu, 2014 b). Une équipe de Speranta TV a parcouru le pays pendant neuf jours pour interviewer en direct, avant le début de chaque conférence, les membres et les invités des églises qui suivaient en direct à l'église le programme l' "Horizon de l'espérance ». Les communautés qui n'avaient pas d'accès par satellite à Speranta TV ont été mobilisées pour se connecter au site internet de la chaîne de télévision ou à la page Facebook, dans leurs églises respectives pour visionner en direct les conférences en ligne. Afin d'encourager leur participation par l'interactivité, les églises ont été invitées à envoyer des photos par e-mail à Speranta TV, des photos qui montraient leurs postures lorsqu'ils regardaient les conférences via internet. De même, tous les spectateurs qui regardaient la série à la télévision, sur Facebook, ou sur le site sperantatv.ro ont été invités à adresser des photos qui, ensuite, étaient projetées durant la transmission en direct et publiées sur la page Facebook dans un album spécialement créé intitulé « Je regarde moi aussi l' " Horizon de l'espérance » (figures 8 et 9) (Dumitrascu, 2014 b). 
Multimédiatisation et événement religieux

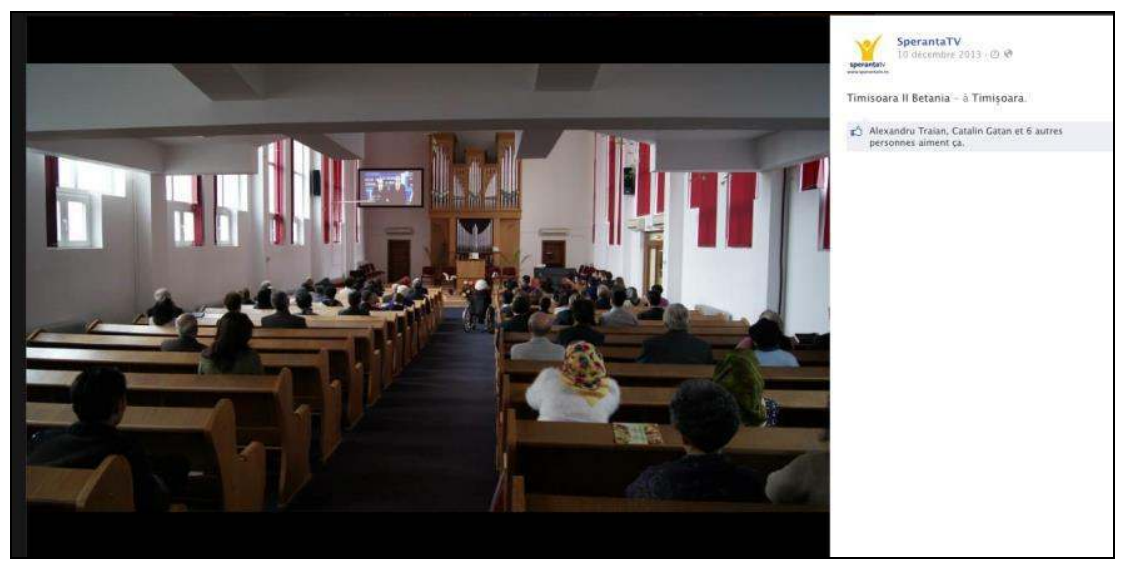

Figure 8. Photo de l'album Facebook « Je regarde moi aussi l'Horizon de l'espérance » (église adventiste Betania de Timisoara).

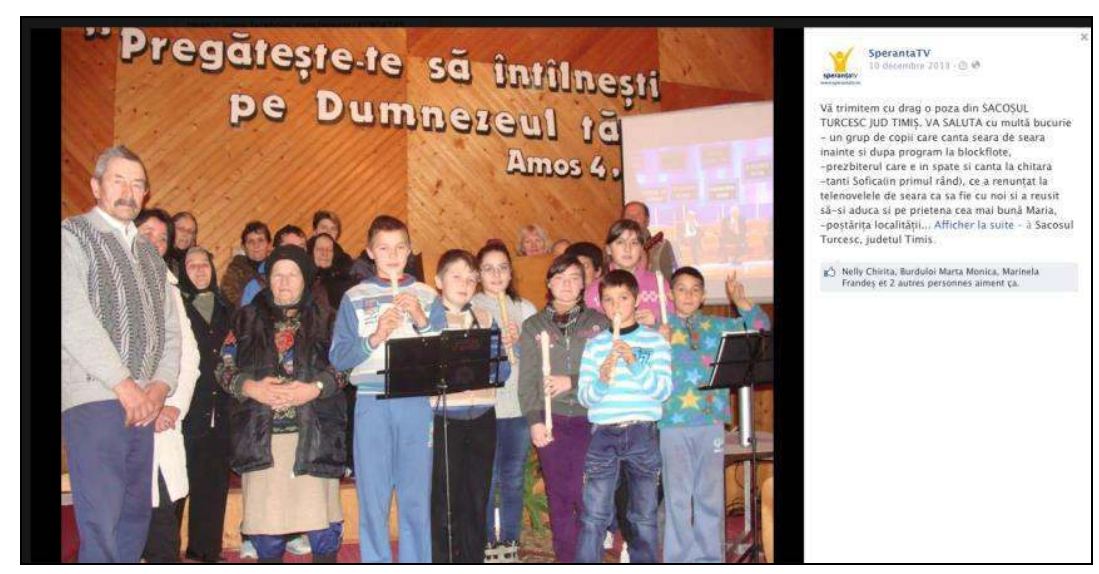

Figure 9. Photo de l'album Facebook « Je regarde moi aussi l'Horizon de l'espérance » (groupe adventiste du village Sacosul turcesc).

L'inclusion du spectateur à travers les images qu'il capte lorsqu'il regarde en direct le programme, soit sur l'écran du téléviseur, soit sur l'écran du vidéoprojecteur, soit sur l'écran du smartphone, soit sur l'écran de l'ordinateur, a le mérite de mettre en exergue de manière directe le rôle interactif du public. II s'agit d'une autoreprésentation du spectateur à travers un dispositif multimédia qui produit du sens par rapprochement de supports et d'écrans multiples et dans le cadre d'une appréhension ubiquitaire-virtuelle de la notion de présence. Ce 


\section{Mihaela Alexandra TUDOR}

dispositif permet aux spectateurs de s'identifier à des protagonistes du programme en participant en direct sur la scène où se produit l'événement sans que leur statut de spectateur soit altéré ou perdu, sans quitter leur scène propre. II s'agit d'un exemple éloquent de multimédiatisation réussie car, au-delà de son côté technologique, la multimédiatisation est un processus de mise en forme des messages, une articulation de différentes composantes du multimédia qui fait naître des mondes interprétatifs, qui organise, combine et crée l'information pour rendre possible la communication (Tudor et Bratosin, 2012, pp.271-280).

Les observations recueillies sur le terrain, le cinquième échantillon, renforcent l'idée que l' « Horizon de l'espérance » a représenté pour Speranta TV l'occasion d'améliorer le dispositif multimédia existant (site web, Facebook, Youtube...) afin d'assurer une couverture large du programme d'évangélisation notamment « par et sur internet, entendu comme un "nouveau carrefour pour l'annonce de l'Evangile (Jean Paul II)" ") (Douyère, 2011, p.152), pour des publics cibles présentant des caractéristiques diversifiées en termes de localisation géographique (pays, ville, village, etc.), de profil démographique, comportemental ou de consommation. II s'agit plus exactement de l'amélioration des prestations du site web Speranta TV et de la création des applications Android et iOS ${ }^{11}$ qui ont été lancées peu avant la campagne d'évangélisation pour faciliter l'accès aux programmes de Speranta TV depuis le portable, la tablette, le smartphone, etc.

Deux campagnes de promotion combinant les médias traditionnels et les nouveaux médias se sont déroulées conjointement avec les activités d'amélioration des prestations. La campagne hors ligne a consisté en 1) des publicités diffusées à la radio Voix de l'espérance, à la télévision Speranta TV et à d'autres petites stations locales; 2) la distribution des prospectus dans tout le pays par les membres des églises locales; 3) la transmission des messages au mégaphone dans plusieurs villes de la partie occidentale du pays; 4) la diffusion des annonces et de la vidéo de 30 secondes avec le message du pasteur-conférencier Doug Batchelor. En ce qui concerne la campagne en ligne, l'attention

11 Systèmes d'exploitation mobile pour les tablettes, smartphones, terminaux mobiles, etc.

tic\&société - 9 (1-2), 2015 


\section{Multimédiatisation et événement religieux}

du public a été dirigée vers internet et les réseaux sociaux. Des informations ont été quotidiennement postées sur le site sperantatv.ro, des bulletins informatifs ont été transmis aux utilisateurs du site adventist.ro, le site officiel de l'Union des églises adventiste roumaine. Quant aux médias sociaux tels que Facebook, Youtube, Twitter, Tumblr et Pinterest, plusieurs ressources diversifiées ont été mobilisées : des liens vers les articles publiés sur sperantatv.ro, des photos depuis le site dougbatchelor.com ou prises dans les coulisses du programme durant la transmission en direct ou envoyées par les téléspectateurs et les internautes regardant le programme, des vidéos quotidiennes annonçant le sujet de la conférence biblique du jour, des liens Youtube avec les chœurs et les chanteurs programmés chaque soir pour ouvrir et clore les conférences, des fragments vidéo des répétitions des chorales, des messages des participants, des statuts annonçant le thème de la conférence du jour ou citant le pasteur Douglas Batchelor.

Ces résultats suggèrent que cette campagne s'est construite autour du modèle de la convergence cross-media avec des tendances, même si elles sont timides, vers le modèle intégré.

La stratégie cross-media de Speranta TV a consisté dans le fait de favoriser des synergies entre les médias différents, déclinant la campagne sur tous les supports disponibles, privilégiant l'interaction entre ces supports et l'interactivité avec les cibles. Par exemple, les prospectus renvoyaient sur le site de la campagne, proposant le téléchargement de la vidéo de présentation et des conférences bibliques, la présentation des livres écrits par Douglas Batchelor ou des liens vers les réseaux sociaux ou vers des sites d'information ou applications sur tablettes et smartphones en liant des personnes à des personnes, ou des groupes d'intérêt à des groupes d'intérêt.

La convergence cross-media facilite une interactivité dont les logiques de fonctionnement peuvent être approfondies à travers la théorie des deux couches de Lev Manovich. Manovich (2001) avance en effet l'idée que les produits des nouveaux médias consistent en deux couches (layers) : la couche de la machine (computer layer) qui est la structure technique du produit, et la couche culturelle (cultural layer) qui suggère que la nature même des nouveaux médias encourage certaines formes d'interaction. II s'agit d'une relation symbiotique dans laquelle les couches culturelle et informatique se déterminent et s'influencent mutuellement et conduisent à de nouvelles formes d'usage des médias et à de nouvelles attentes. Dans le cas de 


\section{Mihaela Alexandra TUDOR}

la campagne l' « Horizon de l'espérance », le chemin entre les données (la couche informatique - vidéos, photos, son, etc.) est la narration interactive (la couche culturelle), c'est-à-dire la manière dont les éléments de l'histoire sont mis en scène : la campagne de télévision devient un objet modulaire, mis à disposition d'un public qui n'est plus figé, statique et « défini » d'emblée. II est défini et "achevé » par l'audience ; il devient partenaire et coproducteur de la campagne d'évangélisation, sa participation active et son concours augmentent considérablement car l'intégration du multimédia et les pratiques et usages du web l'obligent à contribuer lui-même à l'écriture du scénario de la campagne, à être partie prenante de l'histoire. Les téléspectateurs ne sont plus tout simplement invités à se rendre sur le site de Speranta TV pour visualiser une vidéo, ils sont impliqués dans le fait de produire l'histoire en l'enrichissant car ils sont conviés à effectuer des actions telles que transmettre des photos témoignant de leur implication, monter des programmes liturgiques dans leur églises avant la diffusion du direct ou envoyer les enregistrements ou les photos des liturgies pour les présenter en direct ou sur les réseaux sociaux en apportant ainsi de nouvelles dimensions à la campagne.

Ce modèle de mise en réseau des médias du programme l' " Horizon de l'espérance » s'inscrit dans la tendance vers un modèle intégré de convergence média. Le modèle intégré est lié à l'objectif de créer une culture média unique transgressant les limites des médias traditionnels et favorisant une culture de la convergence principalement orientée contenu plutôt qu'orientée plateforme (Aviles et Carvajal, 2008, p.235-236). Les informations deviennent ainsi la matière de base à travailler quel que soit le médium (ibid.). Selon les observations de terrain, l'équipe du programme l' «Horizon de l'espérance » s'est attachée d'une part à utiliser le multimédia qui demande la maîtrise des compétences multiples, et d'autre part à produire du contenu de la même qualité pour tout le multimédia afin d'augmenter la portée de l'information et de la communication. L'observation de terrain a également montré que l'une des principales motivations de l'équipe a été de pouvoir publier du contenu et utiliser tous les médias. Ces résultats sont l'expression d'un processus d'érosion du dévouement de l'équipe à un seul support et média initial utilisé pour renvoyer vers un autre média complémentaire - caractéristique de la convergence cross-media-, sans pour autant considérer la

tic\&société - 9 (1-2), 2015 
Multimédiatisation et événement religieux

convergence comme un but en soi - caractéristique forte du modèle intégré de convergence - (Aviles et Carvajal, 2008). La convergence reste un outil pour la mise en place de ce projet particulier. Toutefois, ce projet pourrait sans doute représenter une occasion pour commencer à mettre en œuvre un modèle intégré de convergence malgré les potentielles difficultés liées à une réalité organisationnelle complexe, à des contraintes structurelles ou à des pratiques coopératives difficiles, caractéristiques à tout type d'organisation.

\section{Conclusions}

Tout comme l'apparition du cinéma n'a pas remplacé le théâtre («Ceci ne tuera pas cela »), la façon de s'engager du média confessionnel Speranta TV avec les NTIC à travers le dispositif multimédia créé pour le programme l' "Horizon de l'espérance » est l'expression que l'évangélisation par les nouveaux médias n'est pas dans une relation autiste ou de rupture avec les médias traditionnels.

Cet article a montré comment la convergence des médias fonctionne dans le cas d'un média confessionnel lors d'une campagne d'évangélisation. La convergence implique généralement différents types de coopération entre plusieurs médias, pas nécessairement de manière intégrée. Le cas étudié suggère une coopération caractéristique au modèle cross-media avec des ouvertures vers le modèle intégré (modèle d'audience active) dans la perspective d'un idéal à atteindre dans l'avenir. Des éléments spécifiques au modèle intégré ont été utilisés par l'équipe, notamment sous la forme inchoative de Transmedia Storytelling qui postule l'engagement intensifié de l'audience dans la réception, dans le déchiffrage, dans le partage et dans l'information autour d'un produit médiatique (Jenkins, 2006), sans pour autant se dévouer à une approche de la convergence de ce genre. Par exemple, pour la mise en œuvre du programme l' " Horizon de l'espérance », il a fallu faire coopérer la télévision classique, internet, les réseaux sociaux, les journaux et revues imprimés (Les signes du tẹmps, Le courrier adventiste - revue et journal mensuels de l'Église adventiste roumaine), la radio, les ouvrages imprimés écrits par le pasteur conférencier (qui pouvaient être achetés par internet 


\section{Mihaela Alexandra TUDOR}

ou aux librairies des éditions Vie et Santé ou acquis suite à un simple appel téléphonique ou e-mail), etc., dans des rapports et logiques différents, ceux d'une collection disparate de pièces assemblées pour remplir un objectif particulier, d'une relation entre les technologies des médias différents, construite de façon encore brute ou improvisée (Jenkins, 2006, p.19).

En se ralliant aux observations d'Andrew Feenberg selon lesquelles «les technologies se stabilisent habituellement après une période où différentes configurations possibles sont en concurrence » (Feenberg, 2013, p.2) ; “ dès qu'elles sont stabilisées, leurs implications politiques et sociales deviennent manifestes » (ibid.), «malgré une évolution sur plusieurs décennies, internet demeure en transition du fait de l'apparition continuelle d'usages innovants » (ibid.), nous espérons que ce cas d'étude pourra faciliter des comparaisons internationales de convergence média pour d'autres médias confessionnels.

\section{Références bibliographiques}

ADORNO T. et M. HORKHEIMER, 1947 [2002], Dialectic of Enlightenment, trad. E. Jephcott, Stanford University Press.

ANGROSINO M.V. et K. A. MAYS de PEREZ, 2000, "Rethinking observation : From method to context", dans N. K. DENZIN et Y.S. LINCOLN (dir), Handbook of qualitative research (2nd ed.), Thousand Oaks, CA, Sage, pp.673-702.

AVILES J.A.G. et M. CARVAJAL, 2008, «Integrated and Cross-Media Newsroom Convergence. Two Models of Multimedia News Production - The Cases of Novotécnica and La Verdad Multimedia in Spain », Convergence: The International Journal of Research into New Media Technologies, vol.14, $\mathrm{n}^{\circ 2}$, pp.221-239.

BABOU I., 1998, «Des discours d'accompagnement aux langages: les nouveaux medias », Etudes de linguistique appliquée, <http://archivesic.ccsd.cnrs.fr/sic_00000066>, dernière consultation le 15 décembre 2014.

BECKER H.S., 2003, "Inférence et preuve en observation participante. Fiabilité des données et validité des hypothèses », 
Multimédiatisation et événement religieux

dans D. CÉFAÏ (dir), L'enquête de terrain, Paris, La Découverte, pp.350-362.

BERGER A., 2000, Media and Communication Research Methods, California, Sage Publications.

BOURDIEU P., 1996, Sur la télévision, Paris, Liber-Raisons d'agir.

BRATOSIN S., M.A. TUDOR et I. COMAN, 2012, "La pratique du sacré dans le World Wide Web: une expérience innovante de la norme », Sciences de la Société, n 81, pp.121-134.

BRATOSIN S., 2014, «Church in the public sphere: production of meaning between rational and irrational », Journal for the Study of Religions and Ideology, vol.13, n 38, pp.3-20.

CAMPBELL H., 2003, "A review of religious computermediated communication research", dans J. MITCHELL et S. MARRIAGE (dir), Mediating religion: Conversations in media, culture and religion, Edinburgh, T \& T Clark/Continuum, pp.213228.

CAMPBELL H., 2006, "Religion and the Internet", Communication Research Trends, vol.26, $\mathrm{n}^{\circ 1}$, pp.3-24.

CAMPBELL H., 2010, When Religion Meets New Media, New York, Routledge.

CANIVET I., 2014, Bien rédiger pour le Web. Stratégie de contenu pour améliorer son référencement naturel, $3^{\mathrm{e}}$ éd., Paris, Eyrolles.

CAREY J.W., 2002, «Preface to the inaugural issue », The Journal of Media and Religion, n¹, pp.1-3.

CASTELLS M., 1999, «Information Age », Radical Philosophy, vol.97, n ${ }^{\circ} 6$, pp.6-17.

CHIRILA I., 2014, « Espace public et "confession" de la foi », dans S. BRATOSIN et M.A. TUDOR (dir), Espace public et communication de la foi, pp.27-35, s.l. [Les Arcs-sur-Argens], larsic.

COBB J., 1998, Cybergrace. The search for God in the digital world, New York, Crown Publishers.

DE IULIO S., 2003, «Lev Manovich, The Language of New Media », Questions de communication, n4, pp.473-475. 


\section{Mihaela Alexandra TUDOR}

DE VRIES H. et S. WEBER (dir), 2001, Religion and Media, Stanford, California, Stanford University Press.

DOUYERE D., 2011, « La prière assistée par ordinateur », Médium, n²7, pp.140-154 <http://www.cairn.info/revuemedium-2011-2-page-140.htm>, dernière consultation le 22 août 2015.

DAWSON L. et D. COWAN (dir), 2004, Religion online: Finding faith on the Internet, New York, Routledge.

DUMITRASCU L., $2014 \mathrm{a}$, « 7 Years of Hope Channel in Romania ", Adventist Review, 22 may, <http://www.adventistreview.org/church-news/\%E2\%80\%8B7years-of-hope-channel-in-romania>, dernière consultation le 29 juin 2014.

DUMITRASCU L., 2014 b, "Tens of Thousands of Eastern Europeans View Internet Outreach", Adventist Review, march, <http://www.adventistworld.org/2014/march/news-briefs/31news-briefs.html>, dernière consultation le 29 juin 2014.

EMERSON R., 2003, «Le travail de terrain comme activité d'observation. Perspectives ethnométhodologistes et interactionnistes », dans D. CÉFAï (dir), L'enquête de terrain, Paris, La Découverte, pp.398-424.

FACEBOOK, Unités de mesure relatives aux publications de la page, <https://fr-fr.facebook.com/help/336143376466063/>, dernière consultation le 7 juin 2014.

FACEBRANDS, 2014, Media sociale in Romania [Médias sociaux en Roumanie], <www.facebrands.ro>, dernière consultation le 23 mars 2014.

FEENBERG A., 2014, "Vers une théorie critique de

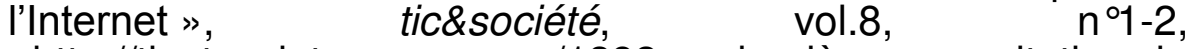
$<\mathrm{http}: / /$ ticetsociete.revues.org/1382>, dernière consultation le 20 juillet 2014.

HELLAND C., 2000, "Online-religion/religion-online and virtual communitas", dans J. K. HADDEN \& D. E. COWAN (dir), Religion on the Internet: Research prospects and promises, New-York, JAI Press, pp.205-223.

HOOVER S.M., 2001, "Religion, media and the cultural center of gravity", dans D. A. STOUT et J. M. BUDDENBAUM 
Multimédiatisation et événement religieux

(dir), Religion and the popular culture: Studies on the interaction of worldviews, Ames, lowa State University Press, pp.49-60.

HOOVER S.M., 2006, Religion in the Media Age, London, Routledge.

HOUSTON D., 1998, Virtual morality, Leicester, Apollos.

INSTITUT NATIONAL DE STATISTIQUE DE ROUMANIE (INS), 2013, Rezultate definitive ale Recensământului Populaţiei şi al Locuinţelor - 2011 (caracteristici demografice ale populaţiei), <http://www.recensamantromania.ro/wpcontent/uploads/2013/07/REZULTATE-DEFINITIVE-

RPL_2011.pdf>, dernière consultation le 26 juillet 2014.

JENKINS H., 2006, Convergence Culture: Where Old and New Media Collide, New York and London, New York University Press.

KATZ E. et P.L. LAZARSFELD, 1955 [2008], Influence personnelle. Ce que les gens font des médias, trad. D. Céfaï, Paris, Armand Colin, Institut national de l'audiovisuel.

LISTER et al., 2009, New Media: A Critical Introduction, New-York, Routledge.

MANOVICH L., 2001, The Language of New Media. Cambridge, Massachusetts, London, England, The MIT Press.

MARION J.-L. 1991, La croisée du visible, Paris, La Différence.

McLUHAN M., 1964, Understanding Media: the Extension of Man, New-York, McGraw-Hill.

O'LEARY S. et B. BRASHER, 1996, "The unknown god of the Internet", dans C. ESS (dir), Philosophical perspectives on computer-mediated communication, Albany, NY, State University of New York Press, pp.233-269.

PALMER M. et J. NICEY, 2012, "Social Media and the Freedom of the Press: a long-term Perspective from within International News Agencies (AFP, Reuters) ", Essachess, Journal for Communication Studies, vol.5, $\mathrm{n}^{\circ 1}(9)$, pp.107-123.

RADIO VOCEA SPERANTEI, <http://www.rvs.ro/desprenoi.html>, dernière consultation le 12 mai 2014.

RUELLAN D. et J. LANGONNE, 2012, "Presse régionale et multisupports", tic\&société, $\quad$ vol.6, $\mathrm{n}^{\circ}{ }^{1}$, 


\section{Mihaela Alexandra TUDOR}

<http://ticetsociete.revues.org/1203>, dernière consultation le 20 juillet 2014.

SERAFEIM K., 2012, "The Impact of Social Media on Press Freedom in Greece: Benefits, Challenges and Limitations ", Essachess, Journal for Communication Studies, vol.5, n¹(9), pp.163-191.

SPERANTA TV, <http://www.sperantatv.ro/>, dernière consultation le 23 juin 2014.

SPERANTA TV, Speranta TV, chaîne de télévision, https://www.facebook.com/sperantatv.ro, dernière consultation le 23 juin 2014.

STOUT D.A., 2011, Media and Religion: Foundations of an Emerging Field, New York, Routledge.

TANASE L.D., 2008, Pluralisation religieuse et société en Roumanie, Bern, Peter Lang.

TRINITAS TV, <https://ro-ro.facebook.com/TrinitasTV>, dernière consultation le 15 août 2014.

TOMKA M., 1998, "Coping with Persecution - Religious Change in Communism and Postcommunist Reconstruction in Central Europe ", International Sociology, vol.13, n², pp.229248.

TUDOR M.A. et S. BRATOSIN, 2013, «Internet and Multimedia: Towards a Theory of Multimediated Figure in Communication Sciences ", dans D. BALABAN, I. HOSU et M. MUCUNDORFEANU (dir), PR Trend, Mittweida, Mittweida Hochschulverlag, pp.271-280.

VLAD T. et L. BECKER, 2012, «Introduction », Essachess, Journal for Communication Studies, vol.5, $\mathrm{n}^{\circ 1}(9)$, pp.7-10.

ZALESKI J., 1997, The soul of cyberspace: How technology is changing our spiritual lives, San Francisco, HarperSanFranciso.

WERTHEIM M., 1999, The pearly gates of cyberspace, London, Virago.

WINKIN Y., 1997, «L'observation participante est-elle un leurre ? », Communication et organisation, n 12, http://communicationorganisation.revues.org/1983, dernière consultation le 14 décembre 2014.

tic\&société - 9 (1-2), 2015 